\title{
Next generation neutrino detectors at the South Pole
}

\author{
Marek Kowalski ${ }^{1, *}$ for the IceCube Collaboration \\ ${ }^{1}$ DESY, Platanenallee 6, D 15738 Zeuthen
}

\begin{abstract}
Since the construction of IceCube was completed in 2010/11, the observatory has delivered several outstanding measurements in neutrino physics and multimessenger astronomy. Additionally, a series of new scientific questions and opportunities appeared. To address these, plans have been developed to expand on the IceCube concept, targeting both lower and higher energies, as well as the central PeV-energy window at significantly improved sensitivity. In this contribution I will outline these plans.
\end{abstract}

\section{Introduction}

Astronomy has explored the Universe through electromagnetic radiation over 20 orders of magnitude in energy, from radio wavelength all the way to X-rays and gamma rays. Yet, at high energies many facets of the Universe remain unobserved as the absorption of the highestenergy gamma rays by the CMB and other radiation fields renders the Universe outside our Milky Way opaque to photons beyond $10^{14} \mathrm{eV}$. With the detection of the first high-energy neutrinos of cosmic origin by the IceCube collaboration in 2013 [1] this window on the highenergy Universe has finally been opened. And the subsequent observation of a source through a neutrino pointing towards a bright, flaring Blazar [2,3] constitutes another success of the exciting field of multi-messenger astronomy. In addition to energy and directional information, the neutrino flavor is currently emerging as an additional, powerful diagnostic. And yet, the current observations constitute only the tip of the iceberg, with many other well-motivated candidate sources within reach of detection and the neutrino production mechanisms still to be understood. Accordingly, the next generation of neutrino detectors are promising a rich harvest in the coming years and decades. A new generation of neutrino detectors are being planed or constructed, such as the Norther detectors KM3NeT in the Mediterranean sea and GVD in Lake Baikal, or IceCube-Gen2 at the South Pole. At low energies, KM3NeT-ORCA and the IceCube Upgrade, with their denser instrumentation, will allow to lower the energy threshold to a few $\mathrm{GeV}$. On the other side of the spectrum, for energies beyond $10^{16} \mathrm{eV}$ the detection of neutrino interactions via the radio signature offers to instrument hundreds of cubic kilometers. Several projects are planned or underway to explore this energy frontier (e.g. ARA/ARIANNA, GRAND) [6]. Together, these detectors provide full coverage of the neutrino sky (North and South) over a very large energy range, with enormously improved sensitivity compared to what is currently available.

In the following, I will focus on the activities at the South Pole, and in particular on the IceCube Upgrade and the IceCube-Gen2 project.

\footnotetext{
*e-mail: Marek.Kowalski@desy.de
} 


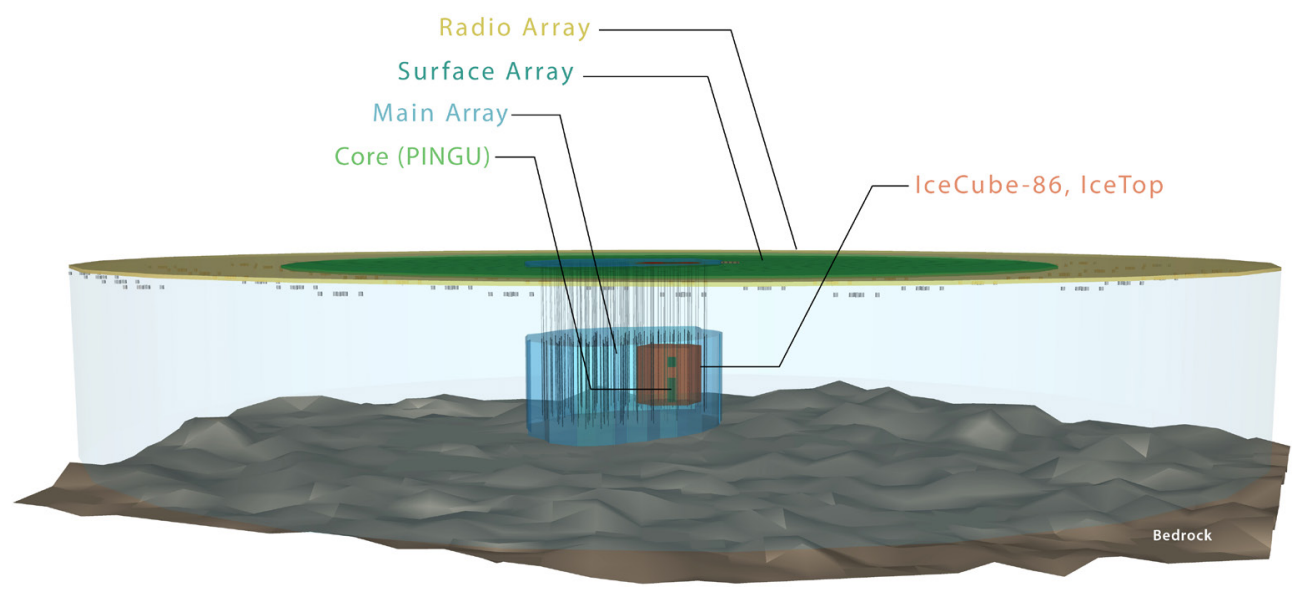

Figure 1. Schematic drawing of the IceCube-Gen2 facility including the main array, a densely instrumented core, a large surface and a radio array. This document focuses on the main array and surface array.

\section{IceCube-Gen2}

IceCube-Gen2, a wide band neutrino observatory $(\mathrm{MeV}-\mathrm{EeV})$ is currently being developed to address the open questions and scientific opportunities that high-energy neutrinos have to offer (see Fig. 1). The scientific goals of IceCube-Gen2 can be summarized as: 1) identify the sources of the highest energy cosmic rays, 2) resolve the complete populations of sources of IceCube's high energy astrophysical neutrinos, 3) decipher the production mechanisms of high energy cosmic particles, 4) obtain a unique multi-messenger view of active galaxies and the explosion of stars, 5) observe the hadronic emission of supernova remnants and other galactic phenomena, 6) study galactic and extra galactic propagation of cosmic rays with neutrinos as tracers and 7) test nuclear, neutrino and BSM physics.

To achieve these goals, IceCube-Gen2 aims to increase the number of observed cosmic neutrinos by ten times compared to IceCube, and be able to detect sources five times fainter than its predecessor. This can be achieved through an IceCube-like geometry with 120 extended strings, a string spacing of $240 \mathrm{~m}$ and a new generation of sensors [4, 5]. One thereby obtains a contained volume that is eight times larger compared to IceCube. If veto criteria are applied as it is the case for a HESE-like analysis, the ratio is even larger. The tradeoffs for a wider string spacing are a degraded angular resolution and a higher energy threshold for separating starting events from the background of penetrating muons. However, for charged current muon neutrino events, the effects on angular resolution are overcompensated (by a wide margin) due to the larger dimensions of the detector and hence larger lever arm in muon reconstruction [5]. The ability for separation of backgrounds in IceCube-Gen2 was tested using IceCube data with a reduced set of strings to mimic the larger string spacing and shown to be sufficient for the targeted energies.

New developments will make the deployment leaner compared to IceCube, e.g. a new generation of sensors that would be narrower in design should allow for narrower drill holes and hence allowing faster deployment and fuel savings. Above $10^{7} \mathrm{GeV}$, the radio technology offers a cost effective alternative for detecting cosmic neutrinos [6]. Hence, in order to expand the reach of the observatory, a radio array is foreseen as an integral component of the IceCubeGen2 facility. 


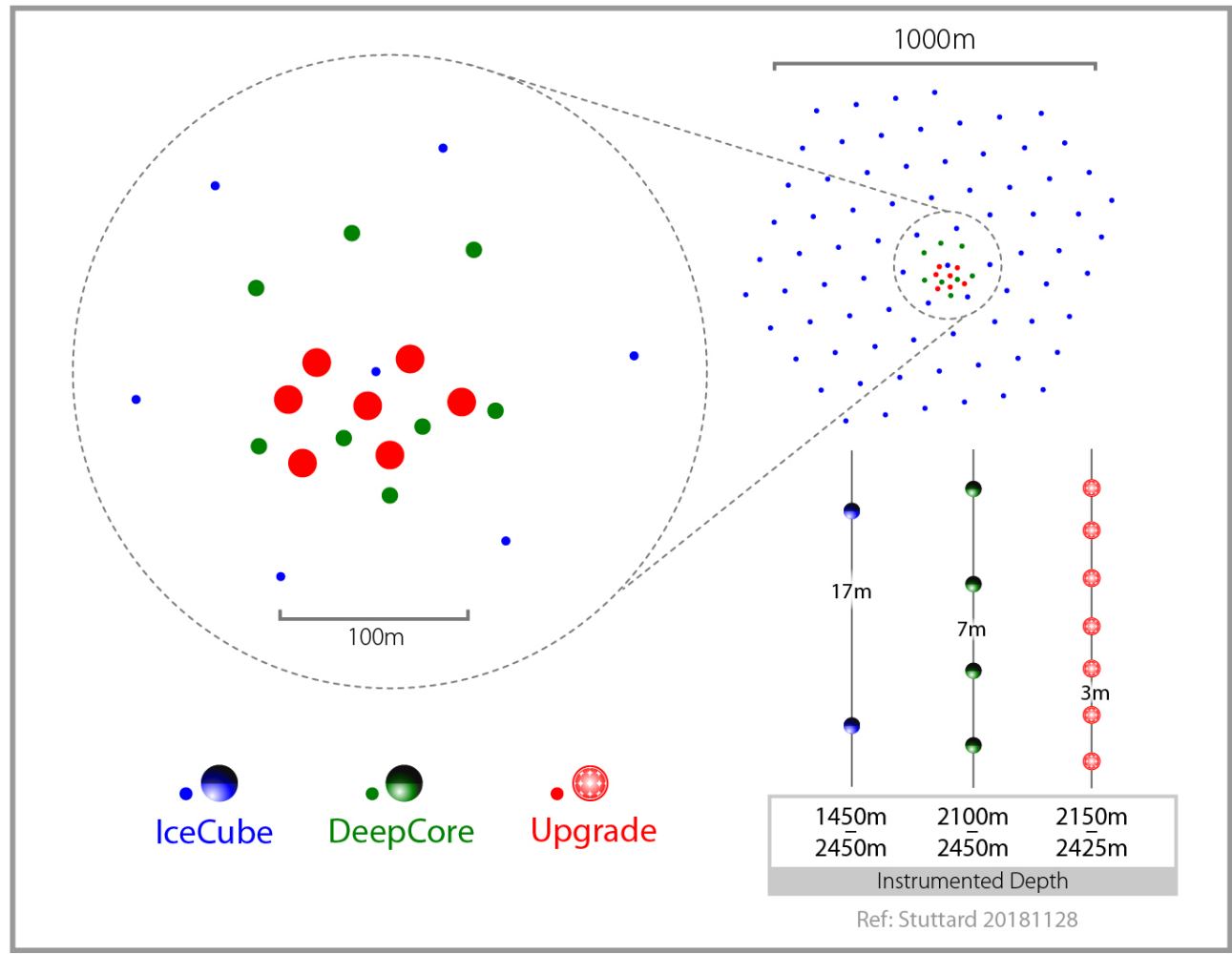

Figure 2. Schematic drawing of the IceCube Upgrade strings, embedded in the IceCube/DeepCore detector.

The goal is to have IceCube-Gen2 operational at the end of the next decade, so that it can fulfill its unique role in shaping the new era of multi messenger astronomy, resolving the numerous mysteries of the high energy universe.

\section{IceCube Upgrade}

The IceCube Upgrade is a mission to improve the existing IceCube/DeepCore detector through new strings with novel, denser instrumentation. This includes in particular new sensors with more uniform acceptance (DEgg containing two 8 inch PMTs [7]) or pixelation (mDOM containing 243 inch PMTs [8]) and a series of calibration sources.

The new strings will increase the light collection in what is already a denser region of the IceCube detector, the DeepCore infill array. By decreasing the separation between IceCube strings from 125 to 75 meters and the distance between light sensors along a string from 17 to 7 meters, DeepCore reduced the energy threshold from the $\mathrm{TeV}$-scale to the $10 \mathrm{GeV}$ scale required for measurements of neutrino properties using atmospheric neutrinos. The seven new strings will be deployed with vertical and horizontal spacings that are another three times smaller than DeepCore, hence lower the energy threshold to GeV scale energies and improve the ability to reconstruct these events. This will boost IceCube's performance at the lowest 
energies and, as a result of this, IceCube will be able to perform measurements of atmospheric neutrino oscillations (including atmospheric tau appearance) with unprecedented sensitivity ${ }^{1}$.

Furthermore the new instrumentation will include advanced calibration devices, for instance POCAM [9] and a new set of cameras, to study the ice properties and sensor characteristics. The improved calibration will not just be applicable to the new strings but for the full IceCube detector, resulting in enhanced reconstruction of events and better particle identification: The cascade signature is the one providing the majority of astrophysical neutrinos identified by IceCube. Cascades currently can be reconstructing with a typical angular resolution of 5-10 degrees, limited by systematic uncertainties. The refined calibration of the ice properties and the existing sensors will enable a reanalysis of more than ten years of archival data and increase the discovery potential of neutrino sources. It will also improve significantly the ability to detect cosmic tau neutrinos. IceCube is sensitive to individual tau neutrino events via their double bang signature, which can be resolved in IceCube in principle to a distance of $20 \mathrm{~m}$ between interaction and decay vertex. The performance for reconstructing events on such "small" distances will be verified through a system of LEDs, that, because of the small spacing of the Upgrade stings, can be made to appear as tau neutrino events near the resolution threshold.

The IceCube Upgrade is funded through the NSF midscale program with a first increment of $\$ 1 \mathrm{M}$ released to set in place the operational and management systems and prepare the final design review that will establish the baseline for the construction funding in early 2019. Of the full project, $\$ 23 \mathrm{M}$ are to be funded through NSF, while the remaining $\sim \$ 10 \mathrm{M}$ that are required to complete the instrumentation are available through external, international contributions. Deployment of the IceCube Upgrade strings is foreseen for the season 2022/23. Beyond the science that the IceCube Upgrade will enable, it will allow testing a new generation of technology and hence serve as a path finder mission for IceCube-Gen2.

\section{References}

[1] M. G. Aartsen et al. (IceCube Collaboration), Science 342, 1242856 (2013) [arXiv:1311.5238].

[2] M. G. Aartsen et al. (IceCube and Fermi-LAT and MAGIC and AGILE and ASAS-SN and HAWC and H.E.S.S. and INTEGRAL and Kanata and Kiso and Kapteyn and Liverpool Telescope and Subaru and Swift NuSTAR and VERITAS and VLA/17B-403 Collaborations), Science 361, no.6389 eaat1378 (2018), [arXiv:1807.08816].

[3] M. G. Aartsen et al. (IceCube Collaboration), Science 361 no. 6398, 147 (2018) [arXiv:1807.08794].

[4] M. G. Aartsen et al. (IceCube Collaboration), arXiv:1412.5106.

[5] J. van Santen (for the IceCube Gen2 Collaboration), PoS ICRC 2017991 (2018)

[6] D. Kostunin, these proceedings.

[7] Y. Makino et al., these proceedings.

[8] L. Classen and A. Kappes, these proceedings.

[9] F. Henningsen et al., these proceedings.

[10] M. G. Aartsen et al. [IceCube Collaboration], J. Phys. G 44, no.5, 054006 (2017) [arXiv:1607.02671].

\footnotetext{
${ }^{1}$ The goals are comparable to that of the PINGU project [10], that follows the same design principles but would foresee a 3 times larger detector with significant sensitivity to the neutrino mass ordering.
} 